\title{
Peertechz
}

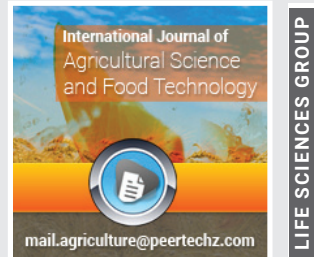

\section{Phenotypic characterization of}

sweet potato (Ipomoea batatas L.) genotypes in Ethiopia for selection of those possessing optimal dual-purpose

Received: 03 February, 2021

Accepted: 06 March, 2021

Published: 08 March, 2021

*Corresponding author: Selamawit Abebe Gitore, MSc, Department of Dry land Agriculture, Faculty of Agricultural Science and Technology, Kenyatta University, Nairobi, Kenya, Tel: +254702601280;

E-mail: abate4fam@gmail.com

Keywords: Sweet potato; Dual-purpose; Morphological characterization; Genotypes; Principal Component Analysis (PCA); Clustering

https://www.peertechzpublications.com

\section{Check for updates}

\section{Selamawit Abebe Gitore ${ }^{1 *}$, Benjamin Danga ${ }^{2}$, Sylvia Henga ${ }^{2}$ and Fekadu Gurmu ${ }^{3}$}

'Department of Dry land Agriculture, Faculty of Agricultural Science and Technology, Kenyatta University, Nairobi, Kenya

${ }^{2}$ Faculty of Agricultural Science and Technology, Kenyatta University, Nairobi, Kenya

${ }^{3}$ Southern Agricultural Research Center, Hawassa, Ethiopia

\section{Abstract}

This research mainly focuses on the morphological characterization of genotypes of sweet potatoes present in Ethiopia for the selection of those with optimum dual-purpose (as food and feed characteristics). From August to December 2019, alpha lattice design using two replication was performed under field condition for this experiment. At 90 days and 180 Days After Planting (DAP), morphological characterization of the 40 sweet potato varieties based on 16 vine, leaf characters and storage root descriptors was performed. In order to exclude those that clearly did not meet the minimum appropriate value of the descriptors provided for selecting dual-purpose cultivars, field observational yield trials were conducted using CIP descriptors. The data generated in this study, using RSTUDIO software, Agricole kit, Partially Balanced Incomplete Block (PBIB), test function, were subject to Variance Analysis (ANOVA). The substantially different mean values were compared using the Tukey test at the $\mathrm{p}<0.05$ stage. Cluster and main component analyses were both conducted using SPSS software (version 20.0). There were major differences among the 40 varieties in almost all of the leaf, vine and root characters examined except Central Leaf Lobe (CLL), Petiole Length (PL) and Storage Root Cortex Thickness (SRCT). Calculations based on root to vine ratio formulas were used to classify dual-purpose use varieties. When the 40 sweet potato genotypes were also tested for dual-purpose use, the analysis found significant phenotypic diversity using the data from the calculation and analyzed data from morphological descriptors. Varieties with R/V values in the $0.2-$ 0.3 range are recommended as dual-purpose high forage varieties, whereas those with R/V values in the $0.3-0.55$ range are graded as dual-purpose high root varieties. As a result, 30 varieties can be recommended as dual-purpose based on the root/vine ratio.

\section{Abbreviations}

DAP: Days after Planting; CIP: Center of International Potato; CLL: Center of Leaf Lobe; PL: Petiole Length; RT: Root Thickness; VID: Vine Internode Diameter; VIL: Vine Internodes Length; PT: Plant Type; MLS: Mature Leaf Size; LLN: Leaf Lobe Number; LVP: Leaf Vein Pigmentation; SYLD: Storage Yield per Ton; RFC: Root Flesh Color; RS: Root Shape; RPC: Root Peel Color; SRCT: Storage Root Cortex Thickness

\section{Introduction}

Sweet potato (Ipomoea batatas (L.) Lam) is a crop for food security and plays a major role in Sub-Saharan Africa and Asia. The crop has high yield and return potential and is easily adaptable to a wide range of farming climates, including in ecosystems and farming systems affected by drought stress than other root crops [1]. In addition to the above advantages, sweet potato storage roots are rich in high-calorie starch, with more amylase than amylopectin, a rich source of dietary fiber, 
minerals, antioxidants, flavonoids, beta-carotene and vitamin A phenolic compounds [2].

Sweet potato is the third most important root crop worldwide after potato and cassava, and ranks sixth in world food crop production (expressed on a dry matter basis) after rice, wheat, potatoes, maize and cassava [3]. However, because of its importance as a food crop, it is ranked fourth in developing countries after rice, wheat, and maize [4]. The crop next to Ensete/false banana (Enseteventricosum (Welw.) Cheesman) is the second best important root crop in Ethiopia $[5,6]$. The crop is widely cultivated in the country in two states, in the area of Debub/southern and Oromia. Production is intensive in the Debub region, mainly in three zones: the Wolayta zone, the Sidama zone, and the Gamo Gofa zone. Above all, the Wolayta and Gamo Gofa zones are well-known for their sweet potato production and the people in these zones are very dependent on sweet potatoes for food security and during the famine period [7]. The majority of smallholder farmers practice mixed farming due to a scarcity of land. These projects provide evidence that the amount of livestock fodder available is inadequate in terms of both quality and quantity [8].

Therefore, dual-purpose sweet potato has become an important crop in maize-based farming systems where it can be planted off-season to meet food safety requirements [9]. Since feed is increasingly scarce, its vines are often used as feed for livestock. High yields, good nutritional quality and low input requirements make sweet potato a potential remedial crop for many rural farmers under marginal conditions [10]. Moreover, in areas where land supply is slowly declining, its usefulness for both food and feed makes it suitable [9]. Sweet potato morphological characterization is performed by assessing differences in the characteristics of the plant, leaf, flower and storage root [9]. This approach was previously used for the identification of sweet potato cultivars, duplicate accessions, detection of specific characteristics and connection with agronomic significance characteristics [5]. For the identification and selection of dual-purpose sweet potato varieties [10], morphological and agronomic characters, such as the storage root to vine ratio, have been used. Agricultural production and productivity were mainly concentrated on cereals in the region, while root crops showed the lowest improvement in seed coverage. Therefore, alternative staple foods, especially sweet potatoes (Ipomoea batatas) have been sought with growing populations and decreasing farm sizes in the region [11]. Neglected/underused crops play a major role in ensuring future food security and can be used directly as alternative crops in the region that are expected to become drought-prone to help farmers generate income and may also be used for further crop development in the breeding program, germplasm resource conservation indirectly.

The main objective of the study is to phenotypic characterization of sweet potato genotypes present in Ethiopia for selection of those possessing optimal dual-purpose characteristics which can be used as a suitable parent for further breeding program.

\section{Materials and methods}

\section{Description of the study area}

The study was conducted At Areka woreda Wolaita Region. The Wolaita Region is one of the 14 SNNPRR Zones (Figure 1). The area's capital city is Sodo and it is situated about $420 \mathrm{~km}$ south of Addis Ababa (Capital city of Ethiopia). Most of the residents belong to the ethnic community of Wolayita, and their language is Wolaytigna, which is the area's main language. Based on altitudes at medium, mid and high altitudes, the

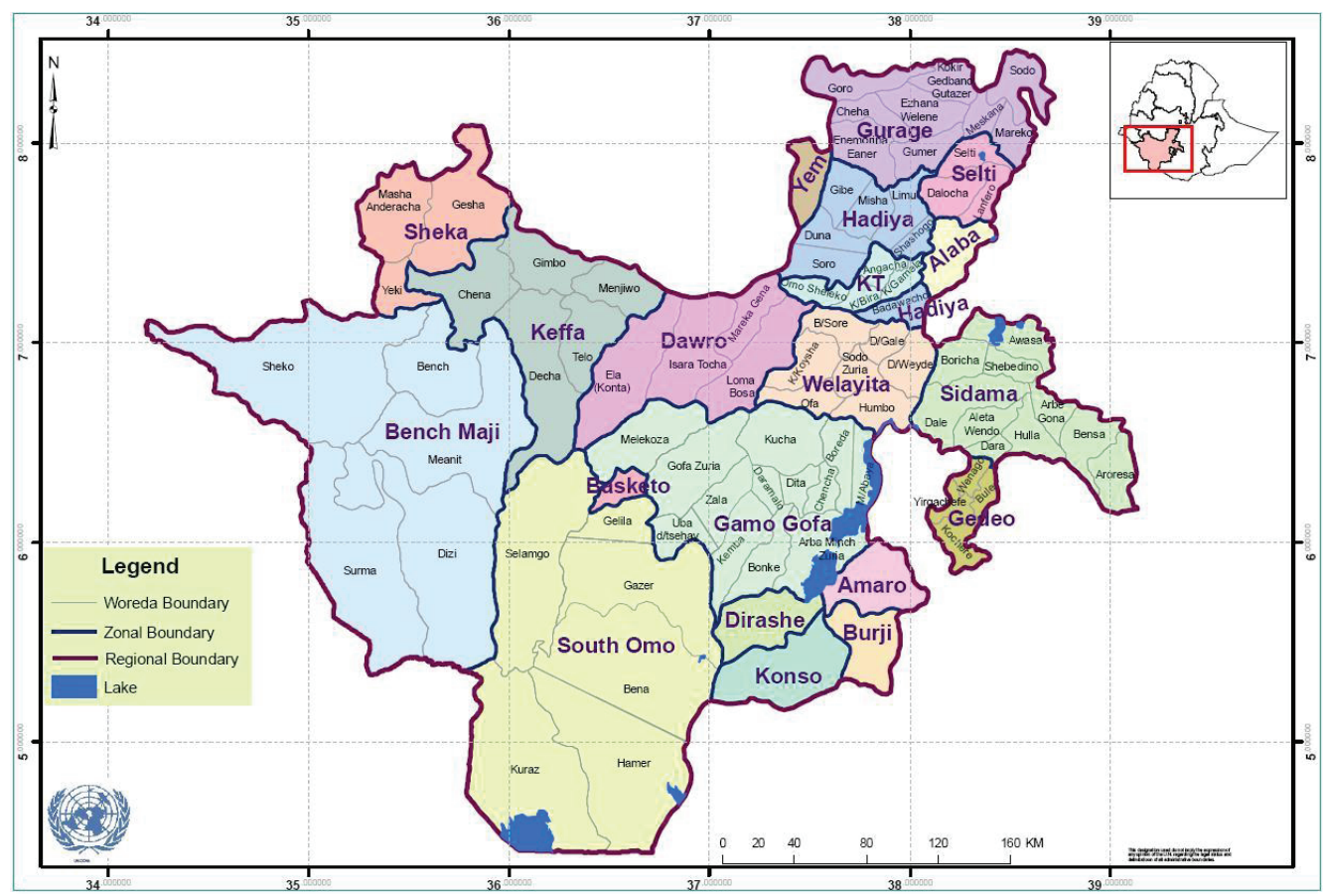

Figure 1: Administrative map of Debub region or South Nations, Nationalities, and Peoples' Region of Ethiopia.

Citation: Gitore SA, Danga B, Henga S, Gurmu F (2021) Phenotypic characterization of sweet potato (Ipomoea batatas L.) genotypes in Ethiopia for selection of those possessing optimal dual-purpose. J Agric Sc Food Technol 7(1): 099-107. DOI: https://dx.doi.org/10.17352/2455-815X.000095 
Wolayita region is stratified. The study area covers a portion of the region between 704 'N37042' E and 704 'N37042' E. The average annual precipitation in the areas of the Wolaita region is about $1300 \mathrm{~mm}$ in the highlands and midlands, respectively. Whereas in the lowland areas is up to $600 \mathrm{~mm}$ or even less. With the highest rains occurring in June-September and short rains in February-March, rainfall is bimodal. More unpredictable and unreliable are the short rains (Belg). In the area, failure of either or both rains triggers shortages. The average daily temperature ranges from $150 \mathrm{C}$ to $300 \mathrm{C}$. The Wolaita region, like the eastern part of Ethiopia, is also heavily affected by drought.

\section{Experimental materials}

For the morphological characterization analysis, forty selected sweet potato accessions that have already been introduced from the CIP Kenya to Ethiopia and germplasm advanced from the crossing experiment performed by the Hawassa Agricultural Research Center [10] in Ethiopia were used Table 1.

\section{Experimental design}

Alpha lattice design with 2 replication under field state was performed. Using $30 \mathrm{~cm}$ spacing between plants and $60 \mathrm{~cm}$ row spacing, genotypes were arranged in 3 rows per plot with 5 plants per row, respectively. Vine tip cuttings were used as planting material and planted 4-6 $\mathrm{cm}$ deep at a length of 30 $\mathrm{cm}$.

\section{Data collected}

At 90 days and 180 days after planting, morphological characterisation of the 40 sweet potato varieties based on 5 vines, 5 leaf characters and 4 storage root descriptors was performed. Except storage root traits such as tuber root shape, storage root cortex thickness, tuber root peel color and storage root flesh color all vine and leaf characters data taken at 90 days after planting (DAP) while the data for storage root traits taken at 180 days after planting (DAP). The international sweet potato descriptors produced by CIP (International Potato Centre) (CIP), AVRDC and IBPGR [12] were used Table 2. In order to exclude those that clearly did not meet the minimum appropriate value of the descriptors provided for selecting dual-purpose cultivars, field observational yield trials were conducted using CIP descriptors.

\section{Calculations for classification of varieties in two dual- purpose}

Classification of dual-purpose use varieties was done based on Leon-Velarde, et al. 2001 [14].

$\mathrm{R} / \mathrm{V}$ ratio calculated using formula $=\mathrm{R} /(\mathrm{R}+\mathrm{V})$

According to classification of Leon-Velarde [13] DP,

1. Forage $(\mathrm{F}), \mathrm{R} / \mathrm{V}<0.2$

2. Dual purpose high forage, DP (F), R/V ranges between 0.2 to 0.3
Table 1: List of the sweet potato genotypes collected for agro-morphological characterization.

\begin{tabular}{|c|c|c|}
\hline Genotype code & Origin/Skin color & Flesh color \\
\hline (Wogabolige)/T34 & purple red & creamy \\
\hline (MUSG014019-7-22)/T16 & cream & Deep orange \\
\hline$(\mathrm{CN} 1448-4926-6) / \mathrm{T} 3$ & pink & Deep orange \\
\hline ( Jane $) / T 18$ & white & creamy \\
\hline (MUSG014052-51-25) /T9 & pink & Deep orange \\
\hline (MUSG014052-51-11)/T15 & pink & deep orange \\
\hline$(\mathrm{CN} 1448-49-28-17) / \mathrm{T} 19$ & cream & intermidate orange \\
\hline (MUSG014052-51-35)/T10 & cream & intermidate orange \\
\hline$(\mathrm{CN} 1448-49-28-8) / \mathrm{T} 31$ & pink & deep orange \\
\hline$(\mathrm{CN} 1448-49-26-7) / \mathrm{T} 28$ & cream & deep orange \\
\hline (Naspot-12)/T39 & pink & intermidate orange \\
\hline (MUSG014046-20-2) /T2 & pink & intermidate orange \\
\hline$(M U S G 014012-26-13) / T 4$ & pink & pale yellow orange \\
\hline (MUSG014052-51-19)/T26 & cream & intermidate orange \\
\hline (MUSG014001-3-11) /T12 & pink & pale yellow orange \\
\hline (MUSG014001-3-12)/T13 & cream & very deep orange \\
\hline$(\mathrm{CN} 1448-49-28-9) / \mathrm{T} 20$ & cream & very deep orange \\
\hline (MUSG014001-3-59)/T6 & pink & pale orange \\
\hline (Ukr/Eju-10)/T35 & white & intermedate orange \\
\hline (MUSG014001-3-11)/T5 & pink & very deep orange \\
\hline (Naspot-13) /T40 & white & intermedate orange \\
\hline (Ukr/Eju-13)/T36 & white & intermedate orange \\
\hline (Carrot C) /T32 & white & cream \\
\hline (Tio Joe-2) /T24 & pink & intermedate orange \\
\hline (MUSG014019-7-50)/T11 & white & intermedate orange \\
\hline$(M U S G 11033-6-1) / T 1$ & cream & deep orange \\
\hline (MUSG014065-21-13)/T21 & cream & deep orange \\
\hline (MUSG014052-51-23)/T14 & cream & deep orange \\
\hline (MGSG1006-7-4)/T30 & white & pale yellow \\
\hline (MUSG014001-3-41)/T8 & cream & intermedate orange \\
\hline (Kabode)/T37 & pink & intermedate orange \\
\hline (Vita)/T38 & pink & intermedate orange \\
\hline (MUSG014001-3-49)/T29 & cream & pale orange \\
\hline (MUSG014019-7-43)/T17 & pink & intermedate orange \\
\hline (M2USG014012-26-32)/T23 & cream & pale yellow orange \\
\hline (MUSG014012-2317-6)/T22 & cream & intermedate orange \\
\hline$($ MUSG014001-3-42) /T7 & pink & intermedate orange \\
\hline (New Kawogo)/T33 & pink & cream \\
\hline (MUSG014065-21-14)/T27 & cream & deep orange \\
\hline (MUSG014019-7-50) /T25 & cream & deep orange \\
\hline
\end{tabular}

3. Dual purpose high root, $\mathrm{DP}(\mathrm{R}), \mathrm{R} / \mathrm{V}$ ranges between 0.3 to 0.55

4. Root $(\mathrm{R}), \mathrm{R} / \mathrm{V}$ ranges $>0.5$.

\section{Statistical analysis}

The data generated in this study was subject to Variance Analysis (ANOVA) using the Agricole package RSTUDIO software.

Citation: Gitore SA, Danga B, Henga S, Gurmu F (2021) Phenotypic characterization of sweet potato (Ipomoea batatas L.) genotypes in Ethiopia for selection of those possessing optimal dual-purpose. J Agric Sc Food Technol 7(1): 099-107. DOI: https://dx.doi.org/10.17352/2455-815X.000095 
Table 2: Vegetative and storage roots characteristics used for evaluation of sweet potato varieties for food and feed use [9]

\begin{tabular}{|c|c|c|}
\hline Morphology & Acceptable description & CIP score \\
\hline $\begin{array}{c}\text { Twining/ } \\
\text { The ability of the vines to climb adjacent } \\
\text { stakes }\end{array}$ & Moderately twining & 5 \\
\hline $\begin{array}{c}\text { Plant Type/ } \\
\text { Growth habit (length of the main vines) } \\
\text { at about } 90 \text { DAP }\end{array}$ & Spreading $(151 \mathrm{~cm}-250 \mathrm{~cm})$ & 7 \\
\hline $\begin{array}{l}\text { Ground Cover/ } \\
\text { Estimated percentage of ground cover } \\
\text { will be recorded }\end{array}$ & High (75\%-90\%) & 7 \\
\hline $\begin{array}{l}\text { Vine Internode/ } \\
3 \text { internode positioned in the middle } \\
\text { section of the vine will be measured. }\end{array}$ & $\begin{array}{l}\text { Intermediate length }(6 \mathrm{~cm} \\
-\quad-9 \mathrm{~cm}) \\
\text { Thick Diameter(10 } \mathrm{mm}- \\
12 \mathrm{~mm})\end{array}$ & 5 \\
\hline $\begin{array}{l}\text { Vine color/ Pigmentation } \\
\text { Pigmentation in the vines. }\end{array}$ & Green & 1 \\
\hline $\begin{array}{l}\text { Vine Apex/ tip pubescence } \\
\text { Hairiness of immature leaves at the tip } \\
\text { will be recorded. }\end{array}$ & Sparse & 3 \\
\hline $\begin{array}{c}\text { Mature leaf shape/ } \\
\text { The leaves situated in the middle section } \\
\text { of the shoot }\end{array}$ & $\begin{array}{l}\text { 1. Rounded, 2.Reniform, } \\
\text { 3. Cordate, and either } 4 \text {. } \\
\text { Triangular }\end{array}$ & $1,2,3$ and 4 \\
\hline Leaf lobe shapes/Leaf lobe number & $\begin{array}{c}\text { No side lobes /Very slender } \\
\text { teeth }\end{array}$ & 0,1 \\
\hline $\begin{array}{l}\text { Mature leaf size/ } \\
\text { The length of } 3 \text { leaves situated in the } \\
\text { central part of the vine will be measured } \\
\text { from the basal to the tip }\end{array}$ & Large $(16 \mathrm{~cm}-25 \mathrm{~cm})$ & 7 \\
\hline $\begin{array}{l}\text { Vein leaf pigmentation/ } \\
\text { The color shown down surface of the } \\
\text { leaves on the veins }\end{array}$ & Green & 2 \\
\hline \multirow[t]{4}{*}{ Storage root } & $\begin{array}{c}1 \text { Tuberous root shape; } \\
\text { Round elliptic, Elliptic, Long- } \\
\text { Elliptic }\end{array}$ & $2,3,8$ \\
\hline & $\begin{array}{l}2 \text { Storage root cortex } \\
\text { thickness -Intermediate } \\
(3 \mathrm{~mm})\end{array}$ & 5 \\
\hline & $\begin{array}{l}\text { (iii)Tuberous root peel color } \\
\text { Any }\end{array}$ & $1-9$ \\
\hline & $\begin{array}{c}\text { (iv)Storage root flesh color } \\
\text { Any }\end{array}$ & $1-9$ \\
\hline
\end{tabular}

The substantially different mean values were compared using the Tukey test at the $\mathrm{p}<0.05$ stage. Some related characters were defined by PCA as the most relevant for classifying the variation within and among the genotypes of sweet potatoes. Morphological parameter cluster studies yielded dendrograms that were used to analyze the phenotypic relationship between the 40 varieties of sweet potato [15].

\section{Results and discussions}

ANOVA was applied on 16 descriptors and showed significant differences of for almost all morphological descriptors. There are significant differences $(\mathrm{p} \leq 0.05)$ in almost all of the vine characters, such as Vine Growth Rate (VGR), Vine Internode Length (VIL), Vine Internode Diameter (VID), plant type (PT), Vine Pigmentation (VIP), Vine Tip Pubescence (VTP), Twining (TW) and Estimated Growth Percentage (EGP).

\section{Vine and leaf characters}

There are highly significant differences $(\mathrm{p} \leq 0.05)$ in almost all of the vine characters, such as Vine Growth Rate (VGR), Vine Internode Length (VIL), Vine Internode Diameter (VID), Plant Type (PT), and Vine Pigmentation (VIP), Vine Tip Pubescence (VTP) and significance difference for the Trait Twining (TW) and Estimated Growth Percentage (EGP). Analysis of variance indicated there are highly significant differences detected for leaf character such as MLS, LVP and LLN. However, CLL and PL were not significantly different $(\mathrm{p} \geq 0.05)$. Among 40 varieties, Twining (TW) was highest in (MUSG014019-7-50) (7.0) and lowest in (MUSG014052-51-11) (0.0), (MGSG10067-4) (0.0),(CN1448-49-28-8)(0.0) and (MUSG014019-7-43) respectively. Vine Internodes Length (VIL) ranged between 8.0 (CN1448-49-26-7) and 2.3 (MUSG11033-6-1). Vine Internodes Diameter (VID) was highest in (MUSG014001-3-41) (10.83), (MUSG014001-3-12) (10.83), (MUSG014019-7-22) (10.83) and lowest in Jane (5.17), (Vita) (5.17) and (MUSG014001-359) (5.17). Plant type (PT) was highest in Naspot-12 (159) and lowest in MUSG014019-7-43 (25) among the varieties. Mature leaf size was highest in (MUSG014012-26-13) (17.5) while MUSG014019-7-43(12.5) had the lowest. Leaf Lobe Number (LLN) ranged between 8.0 and 0.0 (Table 3).

\section{Root characters}

Among the 40 varieties, most of the root characters (Table 4) genotypes had showed highly significant difference ( $\mathrm{p} \leq$ 0.01) for root characters such as Storage Yield per Ton (SYLD), Root Peel Color (RPC), Root Flesh Color (RFC) and significant difference for the trait Root Shape (RS). However storage root thickness (SRCT) was not significantly different. Storage Root Yield Per Ton (SRYLD) was highest in variety MUSG0140013-12 (17.38) while lowest in variety CN1448-49-28-9) (3.18) and (MUSG014001-3-59 (3.18). Root Shape (RS) varied between 9.0 and 2.0, while Storage Root Cortex Thickness (SRCT) was highest in variety MUSG014012-2317-6 (6.75) and lowest in Ukr/Eju-10 (2.45). Among the 40 varieties, Root Flesh Colour (RFC) ranged between 9.0 and 2.0 while it ranged between 8.0 and 1.0 in Root Peel Colour (RPC) (Table 4).

\section{Classification of sweet potato varieties for dual purpose based on $\mathrm{R} / \mathrm{V}$ ratio}

Varieties with a root to vine ratio $(\mathrm{R} / \mathrm{V})$ range of $0.2-0.3$ were classified (class) as high forage varieties, whereas those with a range of $0.3-0.55$ were classified as dual purpose high root varieties Table 5 .

\section{Genetic relations of varieties based on phenotypic cha- racters}

A dendogram based on 16 morphological descriptors of 40 genotypes done to show genetic relations Figure 2. It shows that the varieties separated into two major clusters, A and B. major cluster A is Contained 6 varieties. Super cluster B contained 30 varieties and composed of two sub-clusters I and II, each subdividing further into two other sub-sub-clusters, $\mathrm{Ia}$, Ib, IIa and IIb, respectively. Cluster analysis of the 16 traits revealed that those genotypes in Cluster A had higher values of Plant Type (PT), Vine Internode Length (VIL) And Vine Internode Diameter (VID), Estimated Growth Percentage 
Table 3: Mean score of vine and leaf characteristics of sweet potato varieties under field conditions.

\begin{tabular}{|c|c|c|c|c|c|c|c|}
\hline & & Vine & Characters & & Leaf & Characters & \\
\hline Variety code & $\begin{array}{c}\text { VIL } \\
\text { (score: 1-9) }\end{array}$ & $\begin{array}{c}\text { VID } \\
\text { (score: 1-9) }\end{array}$ & $\begin{array}{c}\text { PT } \\
\text { (score: } 3-9 \text { ) }\end{array}$ & $\begin{array}{c}\text { TW } \\
\text { (score: } 0-9 \text { ) }\end{array}$ & $\begin{array}{c}\text { MLS } \\
\text { (score 0-9) }\end{array}$ & $\begin{array}{c}\text { LVP } \\
\text { (score: } 0-9 \text { ) }\end{array}$ & $\begin{array}{c}\text { LLN } \\
\text { (score: 0-9) }\end{array}$ \\
\hline (Wogabolige) & $7.33 \pm 0.47$ & $10.08 \pm 1.53$ & $151.4 \pm 0.28$ & $6 \pm 1.41$ & $17.3 \pm 1.13$ & $1 \pm 0$ & $3 \pm 2.83$ \\
\hline (MUSG014019-7-22) & $6.33 \pm 0.47$ & $10.83 \pm 0.71$ & $152.6 \pm 1.41$ & $4 \pm 1.41$ & $17.2 \pm 0$ & $6 \pm 0$ & $3 \pm 2.83$ \\
\hline (CN1448-4926-6) & $4.00 \pm 2.36$ & $7.5 \pm 0.71$ & $44.0 \pm 6.22$ & $1.5 \pm 2.121$ & $14 \pm 0.14$ & $1 \pm 0$ & $5 \pm 1.41$ \\
\hline ( Jane ) & $2.85 \pm 1.67$ & $5.17 \pm 0.71$ & $32.9 \pm 4.38$ & $0.0 \pm 0.0$ & $13 \pm 0.71$ & $1 \pm 0$ & $7 \pm 2.83$ \\
\hline (MUSG014052-51-25) & $5.00 \pm .0 .47$ & $6 \pm 0.00$ & $101.3 \pm 32.95$ & $4 \pm 1.41$ & $14.75 \pm 0.35$ & $1 \pm 0$ & $6 \pm 1.41$ \\
\hline (MUSG014052-51-11) & $4.10 \pm .0 .80$ & $6.83 \pm 1.18$ & $44.0 \pm 27.15$ & $0 \pm 0$ & $14.1 \pm 0.14$ & $0 \pm 0$ & $4 \pm 0$ \\
\hline (CN1448-49-28-17) & $3.48 \pm 1.15$ & $6.5 \pm 0.71$ & $53.4 \pm 4.24$ & $1.5 \pm 2.12$ & $13.3 \pm 0.23$ & $1 \pm 0$ & $7.5 \pm 2.12$ \\
\hline (MUSG014052-51-35) & $5.83 \pm 0.24$ & $7.33 \pm 0.47$ & $84.4 \pm 17.25$ & $4 \pm 1.41$ & $13.55 \pm 0.64$ & $0 \pm 0$ & $2 \pm 1.41$ \\
\hline (CN1448-49-28-8) & $6.50 \pm 0.71$ & $10.67 \pm 0.47$ & $157.6 \pm 2.83$ & $0 \pm 0$ & $16.5 \pm 0$ & $1 \pm 0$ & $6 \pm 4.24$ \\
\hline (CN1448-49-26-7) & $8.00 \pm 0.47$ & $7 \pm 0.00$ & $144.9 \pm 4.95$ & $7 \pm 0$ & $14.8 \pm 0.42$ & $3 \pm 0$ & $3.5 \pm 3.54$ \\
\hline (Naspot-12) & $7.00 \pm 0.00$ & $10.5 \pm 0.24$ & $159.10 \pm 2.40$ & $4 \pm 1.41$ & $17.05 \pm 1.48$ & $5 \pm 0$ & $6.5 \pm 3.54$ \\
\hline (MUSG014046-20-2) & $4.00 \pm 0.00$ & $6.5 \pm 0.71$ & $76.6 \pm 21.2$ & $3 \pm 0$ & $14 \pm 0.71$ & $5 \pm 0$ & $4 \pm 4.24$ \\
\hline (MUSG014012-26-13) & $6.83 \pm 0.23$ & $10.67 \pm 0.47$ & $152.6 \pm 1.41$ & $5 \pm 0$ & $17.55 \pm 1.20$ & $1 \pm 0$ & $0.5 \pm 0.71$ \\
\hline (MUSG014052-51-19) & $3.27 \pm 1.79$ & $5.67 \pm 0.94$ & $36.50 \pm 6.50$ & $1.5 \pm 2.12$ & $13.55 \pm 0.78$ & $1 \pm 0$ & $6 \pm 1.41$ \\
\hline (MUSG014001-3-11) & $5.17 \pm 1.65$ & $10.08 \pm 1.53$ & $84.80 \pm 11.88$ & $4 \pm 1.41$ & $14.1 \pm 0$ & $0 \pm 0$ & $4.5 \pm 2.12$ \\
\hline (MUSG014001-3-12) & $3.12 \pm 1.58$ & $10.83 \pm 0.71$ & $27.00 \pm 13.01$ & $0 \pm 0$ & $13.5 \pm 0.71$ & $1 \pm 0$ & $4 \pm 4.24$ \\
\hline (CN1448-49-28-9) & $3.65 \pm 0.92$ & $7.5 \pm 0.71$ & $49.80 \pm 7.07$ & $1.5 \pm 2.12$ & $13.75 \pm 0.35$ & $3 \pm 0$ & $8 \pm 1.41$ \\
\hline (MUSG014001-3-59) & $3.50 \pm .707$ & $5.17 \pm 0.71$ & $64.03 \pm 11.46$ & $1.5 \pm 2.12$ & $13.75 \pm 1.06$ & $4 \pm 0$ & $6.5 \pm 0.71$ \\
\hline (Ukr/Eju-10) & $6.00 \pm 0.00$ & $6 \pm 0.0$ & $152.1 \pm 12.10$ & $6 \pm 1.41$ & $16.95 \pm 1.34$ & $1 \pm 0$ & $7 \pm 0$ \\
\hline (MUSG014001-3-11 & $3.17 \pm .0 .24$ & $6.83 \pm 1.18$ & $49.10 \pm 0.141$ & $1.5 \pm 2.12$ & $13.1 \pm 0.14$ & $1 \pm 0$ & $2 \pm 2.83$ \\
\hline (Naspot-13) & $3.00 \pm 0.00$ & $6.5 \pm 0.71$ & $51.70 \pm 2.69$ & $2.5 \pm 3.54$ & $15 \pm 0$ & $1 \pm 0$ & $5 \pm 5.66$ \\
\hline (Ukr/Eju-13) & $4.75 \pm 0.12$ & $7.33 \pm 0.47$ & $87.10 \pm 15.13$ & $4 \pm 1.41$ & $14.05 \pm 0.07$ & $1 \pm 0$ & $3.5 \pm 4.95$ \\
\hline (Carrot C) & $3.83 \pm 1.65$ & $10.67 \pm 0.47$ & $54.10 \pm 14.00$ & $3 \pm 0$ & $16 \pm 1.41$ & $1 \pm 0$ & $4 \pm 4.24$ \\
\hline (Tio Joe-2) & $5.17 \pm 1.18$ & $7 \pm 0.00$ & $64.60 \pm 2.24$ & $3 \pm 0$ & $14.7 \pm 0.28$ & $1 \pm 0$ & $6.5 \pm 0.71$ \\
\hline (MUSG014019-7-50) & $6.5 \pm 0.71$ & $10.5 \pm 0.24$ & $153.50 \pm 4.38$ & $7 \pm 2.828$ & $16.35 \pm 0.35$ & $1 \pm 0$ & $8 \pm 1.41$ \\
\hline (MUSG11033-6-1) & $2.33 \pm 0.47$ & $6.5 \pm 0.71$ & $43.40 \pm 1.1314$ & $2 \pm 0$ & $13.75 \pm 1.06$ & $0 \pm 0$ & $3.5 \pm 0.71$ \\
\hline (MUSG014065-21-13) & $7.71 \pm 0.24$ & $10.67 \pm 0.47$ & $155.40 \pm 19.2$ & $1.5 \pm 2.12$ & $17.1 \pm 0$ & $1 \pm 0$ & $6 \pm 1.41$ \\
\hline (MUSG014052-51-23) & $4.05 \pm 1.01$ & $5.67 \pm 0.94$ & $75.50 \pm 30.12$ & $2.5 \pm 3.54$ & $13.85 \pm 1.06$ & $2 \pm 1.41$ & $1 \pm 0$ \\
\hline (MGSG1006-7-4) & $3.8 \pm 1.13$ & $10.08 \pm 1.53$ & $56.900 \pm 1.55$ & $0 \pm 0$ & $13.55 \pm 0.64$ & $3 \pm 0$ & $3.5 \pm 4.95$ \\
\hline (MUSG014001-3-41) & $3.5 \pm 0.24$ & $10.83 \pm 0.71$ & $58.700 \pm 0.42$ & $4 \pm 1.41$ & $14 \pm 1.41$ & $4 \pm 0$ & $0 \pm 0$ \\
\hline (Kabode) & $3.1 \pm 1.56$ & $7.5 \pm 0.71$ & $54.10 \pm .42 .43$ & $4 \pm 1.41$ & $14.05 \pm 1.34$ & $1 \pm 0$ & $4 \pm 4.24$ \\
\hline (Vita) & $7.0 \pm 0.47$ & $5.17 \pm 0.71$ & $158.00 \pm 6.79$ & $4 \pm 1.41$ & $16.65 \pm 0.92$ & $1 \pm 0$ & $0 \pm 0$ \\
\hline (MUSG014001-3-49) & $4.33 \pm 2.83$ & $6 \pm 0.00$ & $82.10 \pm 72.27$ & $3.5 \pm 4.95$ & $14 \pm 1.41$ & $1 \pm 0$ & $3 \pm 2.83$ \\
\hline (MUSG014019-7-43) & $3.22 \pm 1.72$ & $6.83 \pm 1.18$ & $25.40 \pm 1.70$ & $0 \pm 0$ & $12.55 \pm 0.4$ & $1 \pm 0$ & $6 \pm 1.41$ \\
\hline (M2USG014012-26-32) & $3.17 \pm 1.18$ & $6.5 \pm 0.71$ & $50.10 \pm 2.97$ & $3 \pm 0$ & $14.8 \pm 0.99$ & $0 \pm 0$ & $8 \pm 1.41$ \\
\hline (MUSG014012-2317-6) & $3.5 \pm 0.24$ & $7.33 \pm 0.47$ & $66.80 \pm 56.57$ & $1.5 \pm 2.12$ & $13.9 \pm 0.14$ & $1 \pm 0$ & $3 \pm 2.828$ \\
\hline (MUSG014001-3-42) & $3.5 \pm 0.24$ & $10.67 \pm 0.47$ & $70.10 \pm 1.27$ & $3 \pm 0$ & $14.5 \pm 1.41$ & $3 \pm 0$ & $4 \pm 4.243$ \\
\hline (New Kawogo) & $4.17 \pm 0.71$ & $7 \pm 0.00$ & $107.20 \pm 11.3$ & $4 \pm 1.41$ & $17.1 \pm 1.56$ & $5 \pm 0$ & $8 \pm 1.41$ \\
\hline (MUSG014065-21-14) & $6.83 \pm 0.24$ & $10.5 \pm 0.24$ & $156.30 \pm 6.65$ & $4 \pm 1.41$ & $15.35 \pm 1.91$ & $1 \pm 0$ & $6 \pm 1.41$ \\
\hline (MUSG014019-7-50) & $2.83 \pm 0.71$ & $6.5 \pm 0.71$ & $30.30 \pm 10.32$ & $2.5 \pm 3.54$ & $14.25 \pm 0.35$ & $1 \pm 0$ & $5 \pm 5.65$ \\
\hline
\end{tabular}

VID: Vine Internode Diameter; VIL: Vine Internodes Length; PT: Plant Type; MLS: Mature Leaf Size; LLN: Leaf Lobe Number; LVP: Leaf Vein Pigmentation. Means followed by the same letters within a column are not significantly different, Tukey's test $(p \leq 0.05)$

(EGP), Vine Pigmentation (VIP), Mature Leave Size (MLS), Twining (Moderately TW), Petiole Length (Intermediate PL), Leaf Vein Pigmentation (LVP), Root Peel Color (RPC) and Root Flesh Color (RFC).

\section{Principal component analysis}

This PCA was performed for all the 16 morphological traits among the 40 sweet potato varieties.PCA revealed 5 principal

Citation: Gitore SA, Danga B, Henga S, Gurmu F (2021) Phenotypic characterization of sweet potato (Ipomoea batatas L.) genotypes in Ethiopia for selection of those possessing optimal dual-purpose. J Agric Sc Food Technol 7(1): 099-107. DOI: https://dx.doi.org/10.17352/2455-815X.000095 
Table 4: Mean score of storage root characteristics of sweet potato varieties under field conditions.

\begin{tabular}{|c|c|c|c|c|c|}
\hline \multirow[b]{2}{*}{ Variety code } & \multicolumn{5}{|c|}{ Storage root characters } \\
\hline & SYLD & SRCT & $\begin{array}{c}\text { RPC } \\
\text { (score: } 0-9 \text { ) }\end{array}$ & $\begin{array}{c}\text { RS } \\
\text { (score: } 0-9 \text { ) }\end{array}$ & $\begin{array}{c}\text { RFC } \\
\text { (score: } 0-9 \text { ) }\end{array}$ \\
\hline (Carrot C) & $8.48 \pm 3.00$ & $5 \pm 1.41$ & $8 \pm 0$ & $5 \pm 0$ & $2 \pm 0$ \\
\hline (Ukr/Eju-10) & $5.30 \pm 1.50$ & $2.45 \pm 0.07$ & $2 \pm 0$ & $2 \pm 0$ & $8 \pm 0$ \\
\hline (CN1448-4926-6) & $12.93 \pm 0.30$ & $5.4 \pm 0.85$ & $6 \pm 0$ & $5 \pm 0$ & $8 \pm 0$ \\
\hline (CN1448-49-28-17) & $3.60 \pm 2.10$ & $5.2 \pm 3.11$ & $1 \pm 0$ & $8 \pm 0$ & $2 \pm 0$ \\
\hline (M2USG014012-26-32) & $11.02 \pm 3.60$ & $4 \pm 2.83$ & $6 \pm 0$ & $2 \pm 0$ & $8 \pm 0$ \\
\hline (Naspot-12) & $5.09 \pm 1.20$ & $5.5 \pm 0.71$ & $6 \pm 0$ & $8 \pm 0$ & $8 \pm 0$ \\
\hline (New Kawogo) & $12.08 \pm 2.10$ & $6.7 \pm 1.84$ & $2 \pm 0$ & $5 \pm 0$ & $7 \pm 0$ \\
\hline (MUSG014052-51-25) & $7.42 \pm 1.50$ & $6 \pm 2.83$ & $2 \pm 0$ & $7 \pm 0$ & $7 \pm 0$ \\
\hline (MUSG014001-3-11) & $9.12 \pm 0.90$ & $5.1 \pm 0.14$ & $6 \pm 0$ & $7 \pm 0$ & $8 \pm 0$ \\
\hline (Vita) & $5.51 \pm 1.80$ & $5 \pm 0$ & $2 \pm 0$ & $5 \pm 0$ & $8 \pm 0$ \\
\hline (Kabode) & $11.87 \pm 1.80$ & $5.5 \pm 3.54$ & $6 \pm 0$ & $5 \pm 0$ & $7 \pm 0$ \\
\hline (MUSG014052-51-19) & $4.24 \pm 0.00$ & $4 \pm 0$ & $6 \pm 0$ & $5 \pm 0$ & $7 \pm 0$ \\
\hline (CN1448-49-28-8) & $7.42 \pm 1.50$ & $5.25 \pm 3.88$ & $6 \pm 0$ & $9 \pm 0$ & $4 \pm 0$ \\
\hline (MUSG11033-6-1) & $9.33 \pm 1.20$ & $7.05 \pm 4.17$ & $2 \pm 0$ & $2 \pm 0$ & $7 \pm 0$ \\
\hline (Wogabolige) & $5.30 \pm 1.50$ & $6.1 \pm 2.69$ & $6 \pm 0$ & $5 \pm 0$ & $4 \pm 0$ \\
\hline (MUSG014001-3-42) & $5.30 \pm 1.50$ & $5.25 \pm 1.06$ & $2 \pm 0$ & $5 \pm 0$ & $9 \pm 0$ \\
\hline (Naspot-13) & $5.09 \pm 1.20$ & $4.55 \pm 0.78$ & $2 \pm 0$ & $7 \pm 0$ & $9 \pm 0$ \\
\hline (MUSG014012-26-13) & $5.09 \pm 1.20$ & $5.6 \pm 3.39$ & $6 \pm 0$ & $5 \pm 0$ & $6 \pm 0$ \\
\hline (MUSG014019-7-50) & $16.32 \pm 2.10$ & $5.25 \pm 3.89$ & $1 \pm 0$ & $5 \pm 0$ & $7 \pm 0$ \\
\hline (MUSG014052-51-35) & $9.96 \pm 2.10$ & $5.7 \pm 3.25$ & $6 \pm 0$ & $6 \pm 0$ & $9 \pm 0$ \\
\hline (CN1448-49-26-7) & $7.63 \pm 1.80$ & $5.75 \pm 4.59$ & $1 \pm 0$ & $8 \pm 0$ & $7 \pm 0$ \\
\hline (MUSG014001-3-11) & $5.09 \pm 1.20$ & $6.05 \pm 0.07$ & $1 \pm 0$ & $7 \pm 0$ & $7 \pm 0$ \\
\hline (MUSG014001-3-12) & $17.38 \pm 0.60$ & $6.15 \pm 1.20$ & $1 \pm 0$ & $5 \pm 0$ & $2 \pm 0$ \\
\hline (MUSG014001-3-41) & $4.66 \pm 0.60$ & $4.65 \pm 0.92$ & $6 \pm 0$ & $8 \pm 0$ & $7 \pm 0$ \\
\hline (MUSG014019-7-22) & $7.42 \pm 1.50$ & $7.6 \pm 1.98$ & $1 \pm 0$ & $5 \pm 0$ & $7 \pm 0$ \\
\hline (MUSG014019-7-43) & $8.48 \pm 0.00$ & $6 \pm 2.83$ & $2 \pm 0$ & $2 \pm 0$ & $8 \pm 0$ \\
\hline (MUSG014019-7-50) & $5.09 \pm 1.20$ & $5.55 \pm 2.19$ & $2 \pm 0$ & $5 \pm 0$ & $8 \pm 0$ \\
\hline (CN1448-49-28-9) & $3.18 \pm 1.50$ & $3.75 \pm 1.77$ & $2 \pm 0$ & $6 \pm 0$ & $8 \pm 0$ \\
\hline (MUSG014001-3-59) & $3.18 \pm 1.50$ & $4 \pm 1.41$ & $1 \pm 0$ & $6 \pm 0$ & $4 \pm 0$ \\
\hline (MUSG014052-51-11) & $5.30 \pm 1.50$ & $4.5 \pm 0.71$ & $2 \pm 0$ & $9 \pm 0$ & $7 \pm 0$ \\
\hline (MUSG014065-21-13) & $9.33 \pm 1.20$ & $5.25 \pm 2.47$ & $6 \pm 0$ & $4 \pm 0$ & $7 \pm 0$ \\
\hline (MUSG014065-21-14) & $9.54 \pm 1.50$ & $5.5 \pm 3.54$ & $6 \pm 0$ & $7 \pm 0$ & $7 \pm 0$ \\
\hline (Ukr/Eju-13) & $3.18 \pm 1.50$ & $3 \pm 1.41$ & $2 \pm 0$ & $7 \pm 0$ & $6 \pm 0$ \\
\hline (MUSG014012-2317-6) & $5.30 \pm 1.50$ & $4.55 \pm 0.64$ & $6 \pm 0$ & $2 \pm 0$ & $7 \pm 0$ \\
\hline (Tio Joe-2) & $11.87 \pm 1.80$ & $6.75 \pm 3.18$ & $2 \pm 0$ & $7 \pm 0$ & $4 \pm 0$ \\
\hline (MUSG014046-20-2) & $4.88 \pm 0.90$ & $2.6 \pm 0.57$ & $2 \pm 0$ & $2 \pm 0$ & $7 \pm 0$ \\
\hline (Jane) & $7.63 \pm 1.80$ & $6.75 \pm 1.77$ & $6 \pm 0$ & $7 \pm 0$ & $7 \pm 0$ \\
\hline (MGSG1006-7-4) & $11.66 \pm 1.50$ & $7 \pm 4.24$ & $6 \pm 0$ & $2 \pm 0$ & $2 \pm 0$ \\
\hline (MUSG014001-3-49) & $5.09 \pm 1.20$ & $5.35 \pm 2.33$ & $2 \pm 0$ & $5 \pm 0$ & $8 \pm 0$ \\
\hline (MUSG014052-51-23) & $5.30 \pm 1.50$ & $4.35 \pm 2.33$ & $2 \pm 0$ & $5 \pm 0$ & $8 \pm 0$ \\
\hline
\end{tabular}

SYLD: Storage Yield Per Ton; RFC: Root Flesh Color; RS: Root Shape; RPC: Root Peel Color; SRCT: Storage Root Cortex Thickness;

Means followed by the same letters within a column are not significantly different, Tukey's test $(p \leq 0.05)$

components (PCs) accounting for $67.7 \%$ of the variation in root, vine and leaf parameters. The first PCA was highly correlated with vine-related traits, such as PT (0.928), VGR (0.922), VIL (0.833) and VID (0.839). The second PC was mainly correlated with root-related traits such as RFC, with correlation values of 0.738 Table 6 .

\section{Conclusions and recommendations}

For the selection of those with optimal dual-purpose characteristics that can be used as a suitable parent for further breeding programs, the research sought to morphologically classify sweet potato genotypes. Dual-purpose sweet potato

Citation: Gitore SA, Danga B, Henga S, Gurmu F (2021) Phenotypic characterization of sweet potato (Ipomoea batatas L.) genotypes in Ethiopia for selection of those possessing optimal dual-purpose. J Agric Sc Food Technol 7(1): 099-107. DOI: https://dx.doi.org/10.17352/2455-815X.000095 
Table 5: List of varieties based on their class for the potential of dual purpose.

\begin{tabular}{|c|c|c|c|c|c|}
\hline Varieties & $\mathrm{R} / \mathrm{V}$ ratio & Class & Varieties & $\mathrm{R} / \mathrm{V}$ ratio & Class \\
\hline (Wogabolige) & 0.36 & $\mathrm{DP}(\mathrm{R})$ & (CN1448-49-28-8) & 0.55 & $\mathrm{DP}(\mathrm{R})$ \\
\hline (MUSG014019-7-22) & 0.30 & DP & (CN1448-49-26-7) & 0.54 & $\mathrm{DP}(\mathrm{R})$ \\
\hline (CN1448-4926-6) & 0.75 & $\mathrm{R}$ & (Naspot-12) & 0.54 & $\mathrm{DP}(\mathrm{R})$ \\
\hline ( Jane ) & 0.24 & $\mathrm{DP}(\mathrm{F})$ & (MUSG014046-20-2) & 0.26 & $\mathrm{DP}(\mathrm{F})$ \\
\hline (MUSG014052-51-25) & 0.75 & $\mathrm{R}$ & (MUSG014012-26-13) & 0.52 & $\mathrm{DP}(\mathrm{R})$ \\
\hline (MUSG014052-51-11) & 0.67 & $\mathrm{R}$ & (MUSG014052-51-19) & 0.64 & $\mathrm{R}$ \\
\hline (CN1448-49-28-17) & 0.69 & $\mathrm{R}$ & (MUSG014001-3-11) & 0.30 & $\mathrm{DP}(\mathrm{F})$ \\
\hline (MUSG014052-51-35) & 0.42 & $\mathrm{DP}(\mathrm{R})$ & (MUSG014001-3-12) & 0.47 & $\mathrm{DP}(\mathrm{R})$ \\
\hline (CN1448-49-28-9) & 0.38 & $\mathrm{DP}(\mathrm{R})$ & (MGSG1006-7-4) & 0.29 & $\mathrm{DP}(\mathrm{F})$ \\
\hline (MUSG014001-3-59) & 0.30 & $\mathrm{DP}(\mathrm{R})$ & (MUSG014001-3-41) & 0.49 & $\mathrm{DP}(\mathrm{R})$ \\
\hline (Ukr/Eju-10) & 0.55 & $\mathrm{DP}(\mathrm{R})$ & (Kabode) & 0.66 & $\mathrm{R}$ \\
\hline (MUSG014001-3-11) & 0.51 & $\mathrm{DP}(\mathrm{R})$ & (Vita) & 0.54 & $\mathrm{DP}(\mathrm{R})$ \\
\hline (Naspot-13) & 0.46 & $\mathrm{DP}(\mathrm{R})$ & (MUSG014001-3-49) & 0.27 & $\mathrm{DP}(\mathrm{F})$ \\
\hline (Ukr/Eju-13) & 0.42 & $\mathrm{DP}(\mathrm{R})$ & (MUSG014019-7-43) & 0.42 & $\mathrm{DP}(\mathrm{R})$ \\
\hline (Carrot C) & 0.73 & $\mathrm{R}$ & (M2USG014012-26-32) & 0.63 & $\mathrm{R}$ \\
\hline (Tio Joe-2) & 0.42 & $\mathrm{DP}(\mathrm{R})$ & (MUSG014012-2317-6) & 0.30 & $\mathrm{DP}(\mathrm{F})$ \\
\hline (MUSG014019-7-50) & 0.55 & $\mathrm{DP}(\mathrm{R})$ & (MUSG014001-3-42) & 0.52 & $\mathrm{DP}(\mathrm{R})$ \\
\hline (MUSG11033-6-1) & 0.66 & $\mathrm{R}$ & (New Kawogo) & 0.88 & $\mathrm{R}$ \\
\hline (MUSG014065-21-13) & 0.41 & $\mathrm{DP}(\mathrm{R})$ & (MUSG014065-21-14) & 0.40 & $\mathrm{DP}(\mathrm{R})$ \\
\hline (MUSG014052-51-23) & 0.25 & $\mathrm{DP}(\mathrm{F})$ & (MUSG014019-7-50) & 0.35 & $\mathrm{DP}(\mathrm{R})$ \\
\hline (CN1448-49-28-9) & 0.38 & $\mathrm{DP}(\mathrm{R})$ & (MGSG1006-7-4) & 0.29 & $\mathrm{DP}(\mathrm{F})$ \\
\hline (MUSG014001-3-59) & 0.30 & $\mathrm{DP}(\mathrm{R})$ & (MUSG014001-3-41) & 0.49 & $\mathrm{DP}(\mathrm{R})$ \\
\hline
\end{tabular}

DP (R): Dual Purpose Root DP (F): Dual Purpose Forage R: Root Only

Table 6: Eigen vectors, Eigen values, total variance and cumulative variance among 11 sweet potato varieties based on 11 morphological characters.

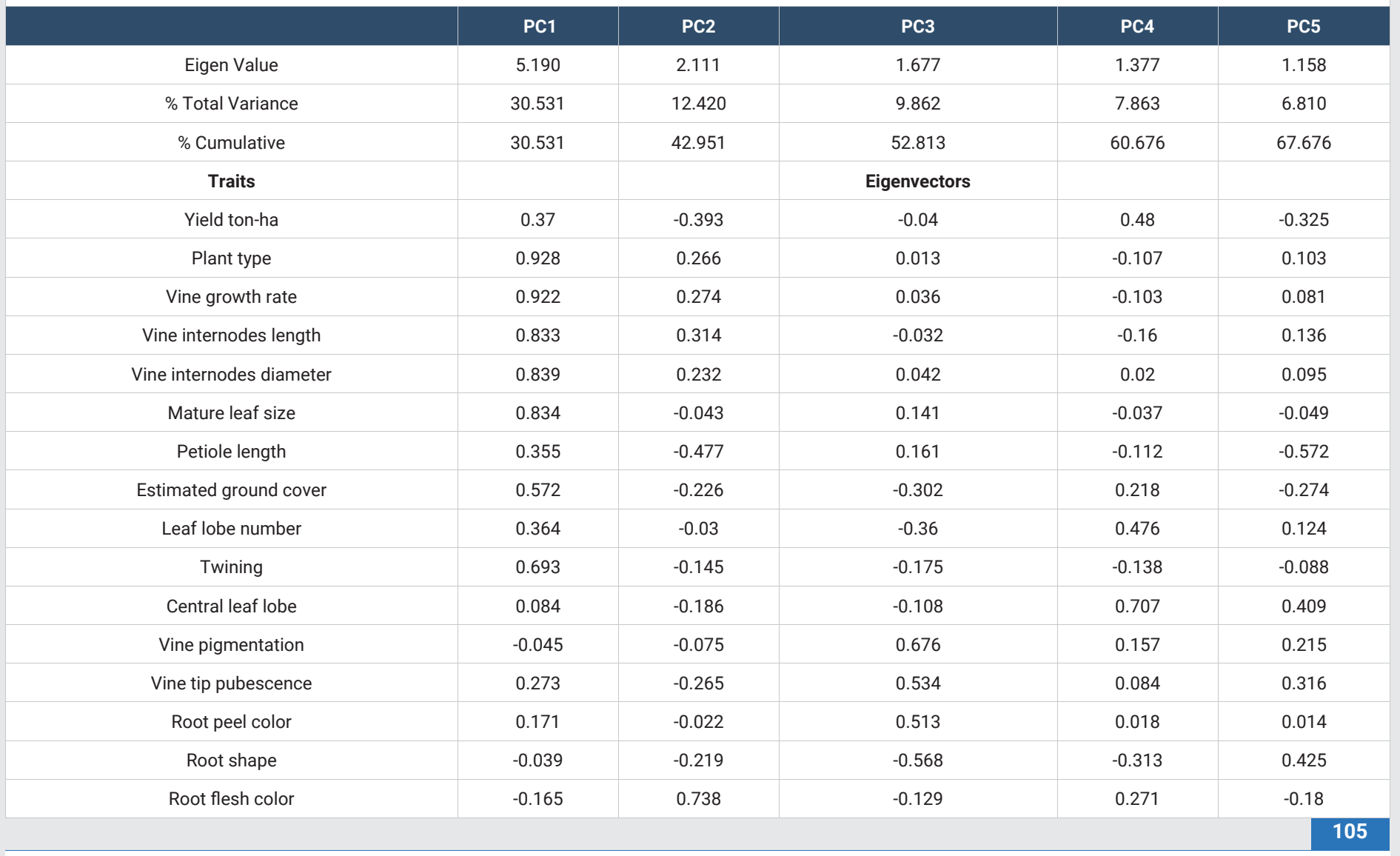

Citation: Gitore SA, Danga B, Henga S, Gurmu F (2021) Phenotypic characterization of sweet potato (Ipomoea batatas L.) genotypes in Ethiopia for selection of those possessing optimal dual-purpose. J Agric Sc Food Technol 7(1): 099-107. DOI: https://dx.doi.org/10.17352/2455-815X.000095 


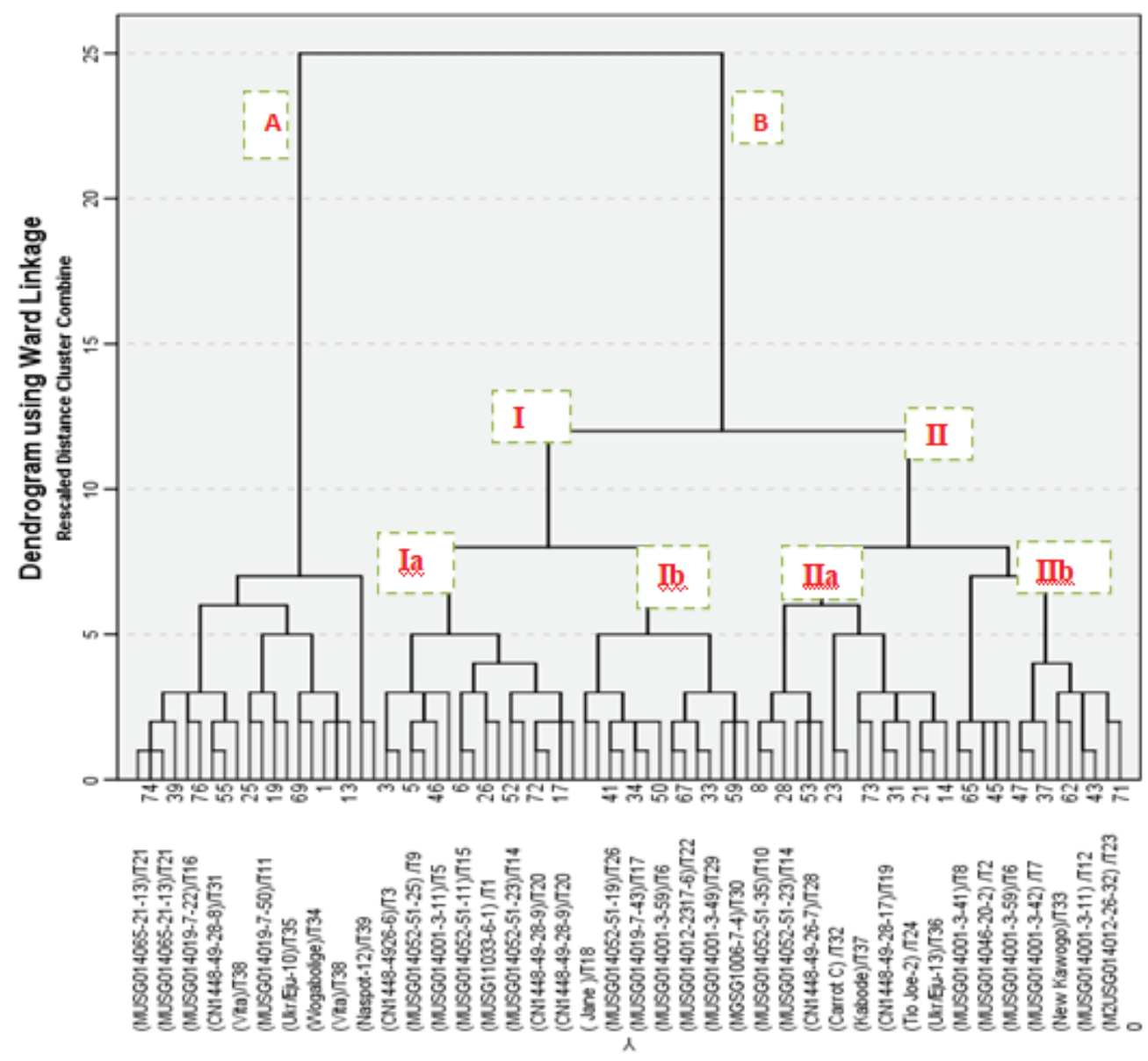

Figure 2: PGMA dendrogram, based on Euclidean distance coefficient, of 40 varieties generated using morphological characters.

allows for a limited number of toppings, so that fodder supplies can be spread throughout the year without dramatically impacting root yields [9]. All spreading species with high ground cover, which is an indication of the availability of fodder, were the varieties selected in this study. With the exception of Petiole length (PL) and Central Leaf Lobe (CLL), all the leaf characters and vine characters evaluated among the varieties were extremely varied. This is because environmental factors affect these characters. The efficient method is the selection of dual-purpose sweet potato varieties based on the root/vine ratio aimed at those varieties that optimize vine production while preserving good storage root productivity [9]. The dendrogram in which varieties with higher values of Plant Type (PT), Vine Internode Length (VIL),Vine Internode Diameter (VID), (Estimated growth cover (EGC), Vine pubescence (VIP) and Mature Leaf Size (MLS), moderately Twining (TW), intermediate Petiole Length (PL), Leaf vein pigmentation (LVP), Root Peel Color (RPC) and Root Flesh Color (RFC) clustered together in major clusters A also showed this variation. The main correlation studies 1 and 2 were the main contributors to the heterogeneity between the varieties. The key traits that led to this variation were TW, PT, EGC, VIL, VID, LLN, CLL, VTP, VGR, MLS, PL, RPC, and RFC. When tested for dual-purpose traits, the analysis found significant phenotypic variation in the 40 genotypes. As a dual-purpose high forage variety, it is recommended when varieties have a 0.2-0.3 range value. Since a variety has a value range of $0.3-0.55$, the high root variety is categorized as a dual purpose. Morphologically, 30 varieties can be suggested as dual-purpose, based on the root/vine ratio. Based on this study, it is proposed that in smallholder crop-livestock operations between humans and livestock, 30 varieties should be grown to help address food vs. feed rivalry. This is because, as feed for animals, the vines and roots of the 30 varieties are suitable and the roots are marketable for direct human consumption. To further classify dual sweet potato varieties using sufficient molecular markers, further studies should be performed.

\section{References}

1. Lebot V (2009) Tropical Root and Tuber Crops: Cassava, Sweet Potato, Yams and Aroids. Economic Botany 64: 86-87. Link: http://bit.ly/20alayA

2. Burri BJ (2011) Evaluating Sweet Potato as an Intervention Food to Prevent Vitamin A Deficiency. Comprehensive Reviews in Food Science and Food Safety 10: 118-130. Link: https://bit.ly/3bpheTq

3. Yada B, Tukamuhabwa P, Wanjala B, Kim DJ, Skilton RA, et al. (2010) Characterization of Ugandan sweetpotato germplasm using fluorescent labeled simple sequence repeat markers. HortScience 45: 225-230. Link: http://bit.ly/2MUPhJI

4. Kays SJ (2005) Sweet potato production worldwide: assessment, trends and the future. Acta Hortic 670: 19-25. Link: http://bit.ly/3v28RF6 
5. Central Statistics Agency (CSA) (2017/18) Crop Production Forecast Sample Survey Report on area and crop production forecast for major grain crops: private peasant holding: Addis Ababa, Ethiopia 25. Link: http://stanford.io/203AtJy

6. Central Statistics Agency (CSA) (2011) Agricultural Sample Survey 2010-11 Report on area and production of major crops: Addis Ababa, Ethiopia 126. Link: https://bit.ly/3kVUD4c

7. Belehu $T$ (2003) Agronomical and physiological factors affecting growth development and yield of sweet potato in Ethiopia. Pretoria University. American Journal of Plant Sciences 6. Link: https://bit.ly/38BfxAH

8. Nyaata OZ, Dorward PT, Keatinge JDH (2000) Availability and use of dry season feed resources on smallholder dairy farms in central Kenya. Agroforestry Systems 50: 315-331. Link: https://bit.ly/2PMjLPn

9. Claessens L, Stoorvogel JJ, Antle JM (2008) Extant assessment of dual-purpose sweet potato in the crop-livestock system of western Kenya: A minimum-data approach. Agricultural Systems 99: 13-22 Link: http://bit.ly/38iHb5b
10. Attaluri S, Janardhan KV, Light A (2010) Sustainable sweetpotato production and utilization in Orissa, India, proceedings. Link: https://bit.ly/3sX66Tz

11. Fekadu G, Hussein S, Mark L (2018) Combining Ability, Heterosis and Heritability of Storage Root Dry Matter, Beta-Carotene and Yield-related Traits in Sweetpotato. HortScience 53: 167-175. Link: https://bit.ly/30n2Xk0

12. Chauvin ND, Mulangu F, Porto G (2012) Food production and consumption trends in sub-Saharan Africa [UNDP Working Paper] Prospects for the transformation of the agricultural sector. Addis Ababa, Ethiopia. Link: https://bit.ly/3kVKa8L

13. Huaman Z (1991) Descriptors for Sweetpotato. International Board for Plant Genetic Resources. CIP(ed). Link: https://bit.ly/3v16Vg1

14. León-Velarde CU (2001) Using competing traits to select dual-purpose sweetpotato in native germplasm. International Potato Center (CIP), Lima Peru 289-294. Link: http://bit.ly/3braEMa

15. Karuri HW, Ateka EM, Amata R, Nyende AB, Muigai AWT, et al. (2010) Evaluating diversity among Kenyan sweet potato genotypes using morphological and SSR markers. Int J Agric Biol 12: 33-38. Link: https://bit.ly/3kUa4cU

\section{Discover a bigger Impact and Visibility of your article publication with}

\section{Peertechz Publications}

\section{Highlights}

* Signatory publisher of ORCID

* Signatory Publisher of DORA (San Francisco Declaration on Research Assessment)

- Articles archived in worlds' renowned service providers such as Portico, CNKI, AGRIS, TDNet, Base (Bielefeld University Library), CrossRef, Scilit, J-Gate etc.

* Journals indexed in ICMJE, SHERPA/ROMEO, Google Scholar etc.

* OAI-PMH (Open Archives Initiative Protocol for Metadata Harvesting)

* Dedicated Editorial Board for every journal

* Accurate and rapid peer-review process

* Increased citations of published articles through promotions

* Reduced timeline for article publication

Submit your articles and experience a new surge in publication services (https://www.peertechz.com/submission).

Peertechz journals wishes everlasting success in your every endeavours.

Copyright: @ 2021 Gitore SA, et al. This is an open-access article distributed under the terms of the Creative Commons Attribution License, which permits unrestricted use, distribution, and reproduction in any medium, provided the original author and source are credited.

Citation: Gitore SA, Danga B, Henga S, Gurmu F (2021) Phenotypic characterization of sweet potato (Ipomoea batatas L.) genotypes in Ethiopia for selection of those possessing optimal dual-purpose. J Agric Sc Food Technol 7(1): 099-107. DOI: https://dx.doi.org/10.17352/2455-815X.000095 